International Journal of Advanced Technology in Mechanical, Mechatronics and Materials

(IJATEC)

Vol. 02, No. 1 (2021) 11-21

Institute for Research on Innovation and Industrial System (IRIS)

\title{
Two Dimensional CFD Analysis and Flow Optimization of Transmission Cooling Scoop for Longitudinal Powertrain Applications
}

\author{
Jacob Viertel ${ }^{a^{*}}$, Rachmadian Wulandana ${ }^{a}$ \\ aDepartment of Engineering, State University of New York at New Paltz, New York, United States
}

\begin{abstract}
Two dimensional finite area method simulation was conducted to optimize the convective cooling performance of a transmission cooling scoop for longitudinal vehicle powertrain applications. Cooling of the transmission in an automobile is important to prevent premature wear or sudden failure caused by prolonged overheating of internal transmission components. The most common method for transmission cooling requires a small energy input for powering a pump to cool the transmission by circulating transmission fluid through a heat exchanger. An alternative cooling method was designed utilizing a simple scoop geometry to induce forced convection from ambient air to cool the transmission with no energy input requirement. Two dimensional simulation of this alternative cooling method was conducted in ANSYS Fluent. Fluid flow and heat transfer performance were analyzed for three proposed cooling scoop designs. Further flow optimization was achieved with parametric study regarding angle at which the cooling scoop is positioned relative to the transmission. Three dimensional simulation was conducted for improved observation of the physical model. Based on the simulation results, optimal geometry and future design improvements have been determined. A peak simulated heat transfer of $11.14 \mathrm{~kW} / \mathrm{m}^{\wedge} 2$ was achieved with scoop angle of 45 degrees. Future research investigating the effects of induced turbulence to improve convective heat transfer would be beneficial.
\end{abstract}

Keywords: cooling; forced convection; automotive; drivetrain

DOI: $10.37869 /$ ijatec.v2i1.39

Received 22 December 2020; Accepted 28 January 2021; Available online 19 April 2021

(C) The Authors. Published by IRIS. This is an open access article under the CC BY-NC-SA license cc) (1)(2)

\section{Introduction}

When driving at high speeds for a long duration of time, the transmission within an automobile can become very hot. This is a result of the heat generated by friction between the meshing gears within the transmission. Normally, the fluid within the transmission helps to lubricate the gears and reduce this friction and prevent overheating. However, as vehicle transmissions become more advanced with increased moving components, the heat generated by friction becomes more of an issue. Prolonged operation can generate enough heat to reduce the viscosity of the transmission fluid and consequently increase the friction between the gears [1]. This can cause premature wear and ultimately damage the transmission [2] [3]. Hence, research focusing on preventing the wear and failure of vehicle powertrain components such as the transmission have become a popular area of study. Some manufacturers have even developed failsafe systems that monitor the transmission temperature. Normal transmission operating temperature exists at approximately $79.4^{\circ} \mathrm{C}$ [4]. When overheating occurs over $150^{\circ} \mathrm{C}$, the failsafe system reduces engine output power in an attempt to prevent damage [2]. To prolong the life of the transmission and prevent premature and possibly sudden failure due to overheating, additional cooling of the transmission is necessary. Many modern 
vehicles, especially in high performance applications, are equipped with cooling systems utilizing an air to liquid heat exchanger and a pump which circulates and cools the transmission fluid [5]. An example of this type of system is found in US Patent 5678461. In this system designed by Alan C. Stine, transmission fluid is cooled with an air to liquid heat exchanger mounted beneath the transmission. Transmission fluid is circulated through the heat exchanger using a pump driven by the motor of the vehicle as an accessory belt component [6]. Heat is first transferred from the transmission to the fluid. The fluid then circulates through the heat exchanger where heat is transferred to the ambient air below the transmission. This type of cooling has in many cases shown to efficiently maintain a safe maximum operating temperature within the transmission. However, there are a couple drawbacks of this method. This specific design puts the transmission at risk of losing fluid through the external fluid lines leading to and from the heat exchanger. If any of the fluid lines become damaged from road debris, the transmission could lose lubrication and fail catastrophically. Secondly, this type of system requires an energy input to power the pump. This consequently puts an additional load on the engine which reduces fuel efficiency.

A similar design in US Patent 4945981, utilizes the same concept but positions the heat exchanger at the front of the vehicle along with the radiator and A/C condenser. In this design by Shrikant Joshi, a heat exchanger is again used to cool transmission fluid circulated by a pump driven by the engine. However, in this case the heat exchanger is not mounted to the bottom of the transmission but is instead mounted in front of the vehicle with the engine radiator and A/C system condenser [7]. By relocating the heat exchanger to the front of the vehicle where it is better protected by the grill and radiator support, this design reduces the risk of puncturing fluid lines from road debris. Again, the biggest drawback to an oil cooling system like this is the input energy requirement of the pump, fulfilled by the vehicle engine. This additional load on the engine comes at the cost of decreased engine performance and efficiency.

A potential alternative for transmission cooling which requires no input energy is cooling by forced convection using the ambient air at high vehicle speeds. This method of cooling is commonly used to cool vehicle brake systems. This method has been optimized for better cooling performance with the addition of vents in brake disks to direct air directly into the disks [8]. This method can be applied to transmission cooling by utilizing the relative bulk motion of ambient air when a vehicle is moving at high speeds to cool the transmission via forced convection. In this application, cool ambient air is redirected with a scoop to the higher temperature transmission housing. Heat energy is then transferred from the transmission to the ambient air through forced convection. This method eliminates the risk of leaking transmission fluid through external fluid lines and most importantly does not require any input energy. An example of a transmission cooling design utilizing this forced convection cooling method is US Patent 4848453. This design intakes air from below the vehicle and redirects it to the transmission housing using rectangular slanted ducts. Heat is then transferred from the transmission to the moving air through forced convection. This method helps to cool the transmission without any energy input [9]. The only compromise with this specific design is that the vehicle's ground clearance is reduced by 8 inches with the addition of this scoop. This may be suitable for large trucks and SUVs, but for low ground clearance vehicles like sedans and sports coupes, this design may not fit beneath the vehicle and if so, it would greatly increase the risk of bottoming out over uneven road surfaces or speed bumps. A more compact design would be needed to prevent the risk of bottoming out and damaging the underneath of the vehicle.

The use of other geometries like circular ducts have been implemented for this type of cooling as well. In this type of system, a circular inlet is positioned towards the front of the vehicle where air can easily enter. This air is then directed through a system of circular ducts that lead to the transmission housing where the air finally exits for convective cooling of the transmission [10]. The problem with this system is that it is often complex and requires a specific design for each application.

A transmission cooling system that can maintain safe transmission operating temperatures without the need for an energy input would be favorable in allowing for reduced load on the engine and improved fuel efficiency. Transmission cooling via forced convection can achieve this by utilizing ambient air at high vehicle speeds, however this method typically requires large obstructing devices that reduce vehicle ground clearance. Hence, this study focused on the optimization of a low profile transmission cooling device utilizing forced convection by ambient air. Cooling by forced convection with a compact, low profile transmission cooling scoop would allow for an easy to produce, low cost 
solution for preventing transmission overheating and premature failure without an energy input requirement.

\section{Equations and Theory}

Transmission cooling by forced convection relies heavily on maximizing convective heat transfer by optimizing fluid flow. The cooling process in this type of transmission cooling system is illustrated in the labeled graphic in Figure 1.

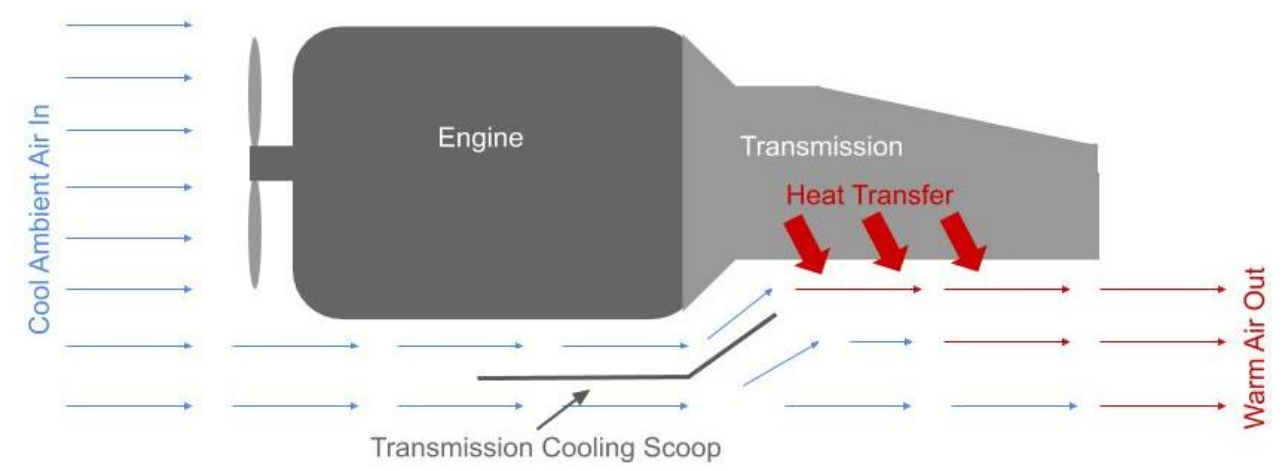

Figure 1. Convective heat transfer induced by cooling scoop

As seen in Figure 1, cool ambient air moves around the engine and transmission of the vehicle with speeds relative to the motion of the vehicle. This ambient air velocity is typically turbulent. For this study, the average velocity of the fluid is assumed to be 24.6 meters per second in the horizontal direction. This was chosen as it is equivalent to many typical United States highway speed limits (55 miles per hour). As this cool ambient air travels below the engine, the geometry of the cooling scoop forces some of the air to change in direction and flow towards the transmission. This increase in the bulk motion of air flowing around the transmission causes forced convective heat transfer to occur. The induced forced convection allows for more rapid heat transfer from the high temperature transmission to the lower temperature air, effectively cooling the transmission quicker. This induced convective heat transfer is illustrated in Figure 2.

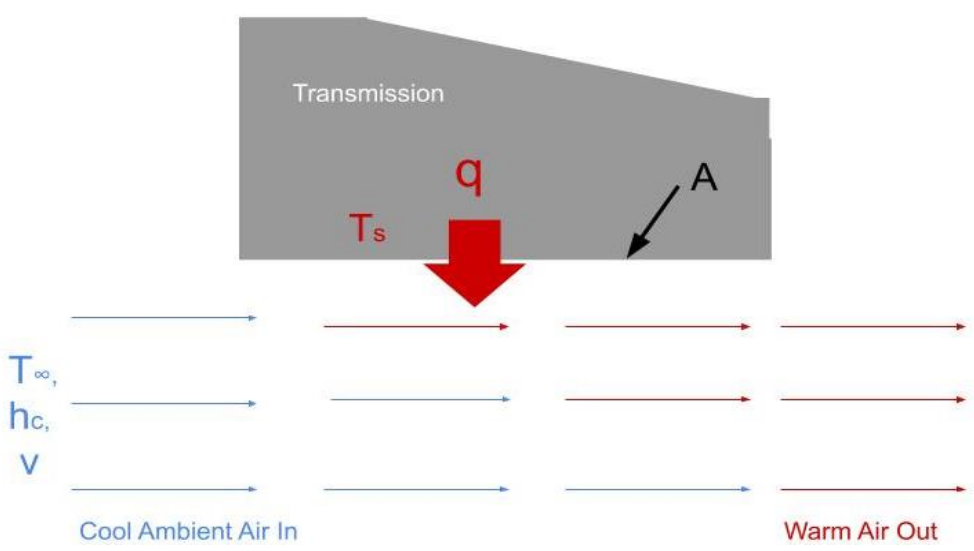

Figure 2: Forced Convection Between Transmission and Ambient Air Bulk Motion

The rate of convective heat transfer from the transmission to ambient air can be calculated using Newton's Law of cooling, Equation (1).

$$
q=h_{c} * A *\left(T_{s}-T_{\infty}\right)
$$

The heat transferred per unit time, $q$, is equal to the product of the convection coefficient, hc, exposed surface area of the transmission, $\mathrm{A}$, and temperature differential, where $\mathrm{T}_{\mathrm{s}}$ is the surface temperature of the transmission, and $\mathrm{T}_{\infty}$ is the initial temperature of the incoming ambient air. To optimize the 
rate of heat transfer between the transmission and the ambient air without having control over the temperature differential, either the exposed area or convection coefficient can be increased. Most transmissions already utilize cooling fins integrated into the bottom of the transmission housing for increased surface area to improve heat transfer. This leaves one more factor to improve, the convection coefficient. The convection coefficient is dependent on the speed of the bulk motion of fluid, in this case ambient air. If the temperature differential and exposed surface area are assumed to be constant, the convection coefficient for ambient air can be approximated using Equation (2). This equation is based on interpolation of air flow data from a study conducted in 2018 [11].

$$
h_{c, \text { air }}=13.4712 * v^{0.789}
$$

If the fluid velocity, $\mathrm{v}$, of ambient air bulk motion is known in meters per second ( $\mathrm{m} / \mathrm{s}$ ), the equivalent convective coefficient, $h_{c}$,air can be approximated in watts per square meter times kelvin $\left(\mathrm{W} / \mathrm{m}^{\wedge} 2^{*} \mathrm{~K}\right)$. Equation (2) is shown graphically in Figure 3.

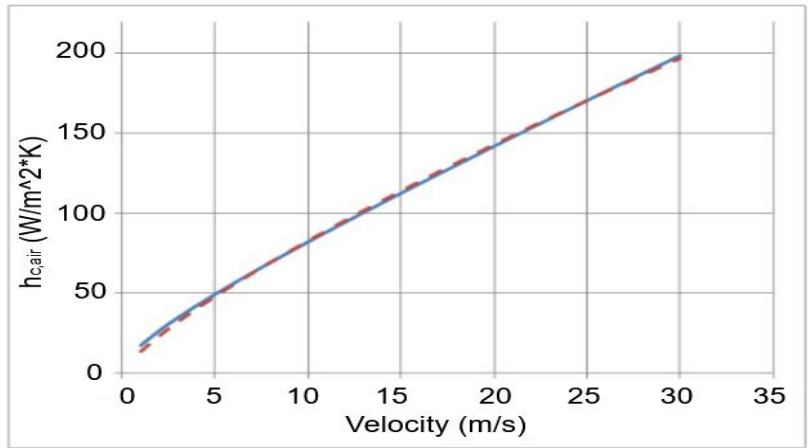

Figure 3. Air Flow Velocity vs. Convection Coefficient [11]

From the plot of Equation (2), it is observed that increasing air flow velocity results in a larger convection coefficient and consequently a greater heat transfer rate. Achieving the largest possible air flow velocity directed towards the transmission would generate the greatest potential for heat transfer between the transmission and ambient air. This is what the cooling scoop aims to accomplish. The velocity of air exiting the tip of the cooling scoop becomes of interest creating a fluid dynamics optimization problem involving flow around an immersed body. Flow around an immersed body is relatively complex and therefore is typically analyzed through physical experimentation or computer simulation. This study utilizes the finite volume method in ANSYS Fluent to analyze the change in velocity from air entering the cooling scoop to the air exiting the cooling scoop tip directed towards the transmission. The ideal cooling scoop geometry would result in the greatest increase in air velocity, in turn yielding the largest convection coefficient and heat transfer rate.

\section{Research Methodology}

This analysis focused on the optimization of a forced convection method transmission cooling system utilizing compact scoop geometry. Three compact transmission cooling scoop designs were created for analysis and optimization. The main requirement for these three designs was to effectively redirect air to the transmission while remaining as compact as possible to minimize reduction in vehicle ground clearance. The cross section profile of each of the three designs were sketched and dimensioned in 2D, shown in Figure 4.
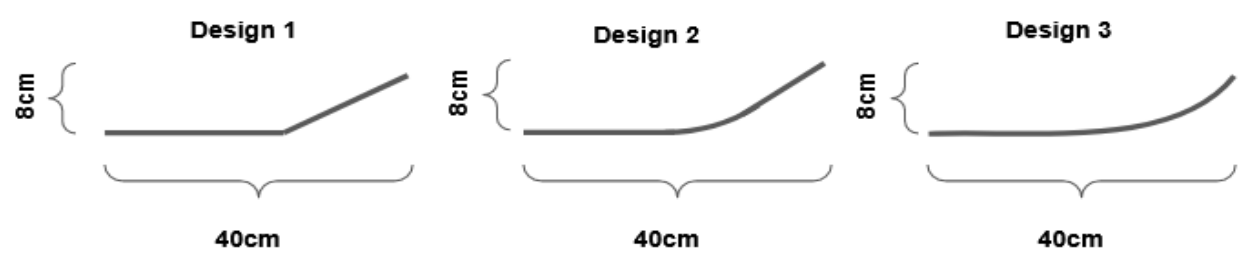

Figure 4. Initial design sketches for transmission cooling scoop 
All three designs are assumed to have a thickness of $2 \mathrm{~mm}$ and are scaled within a $40 \mathrm{~cm}$ by $8 \mathrm{~cm}$ constraint to reduce the decrease in ground clearance when mounted to a vehicle. The first design shown to the left consists of two $2 \mathrm{~mm}$ thick plates at an angle of 28 degrees. The second design shown in the middle is similar to the first design but with a curved transition from the horizontal plate to the diagonal plate. The last design shown to the right consists of one $2 \mathrm{~mm}$ plate with a constant radius of curvature throughout. The physical three dimensional design would have a width of $30 \mathrm{~cm}$ and added side walls at each edge. Since all three designs share equal features in their width and sidewall geometry, the simulation can be simplified to a two dimensional CFD analysis of the cross section profiles. While the majority of the analysis is focused on the optimization of the simplified two dimensional designs, a three dimensional design was also created and simulated for the purpose of observation of the physical product.

Each of the three transmission cooling scoop designs were modeled in 2D ANSYS SpaceClaim as shown in Figure 5.

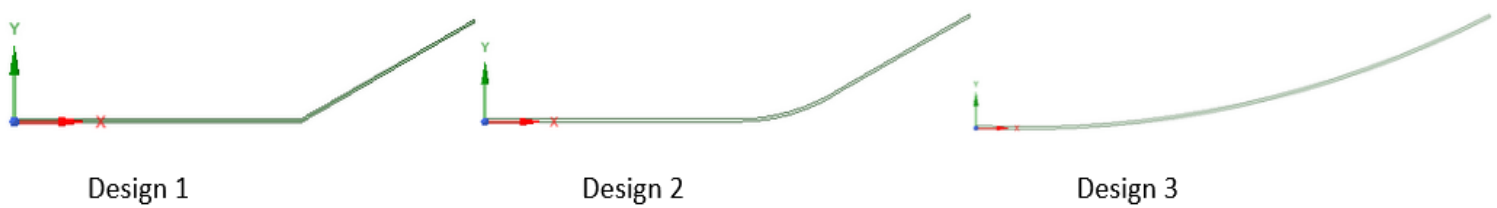

Figure 5. Transmission cooling scoop designs in SpaceClaim

To simulate the flow of air around the cooling scoop designs, a geometry had to be created to represent the surrounding fluid. This was done with a simple $2 \mathrm{D}$ rectangular surface scaled ten times larger than the cooling scoop. The rectangular fluid geometry modeled for cooling scoop design 1 is shown as the green surrounding area in Figure 6.

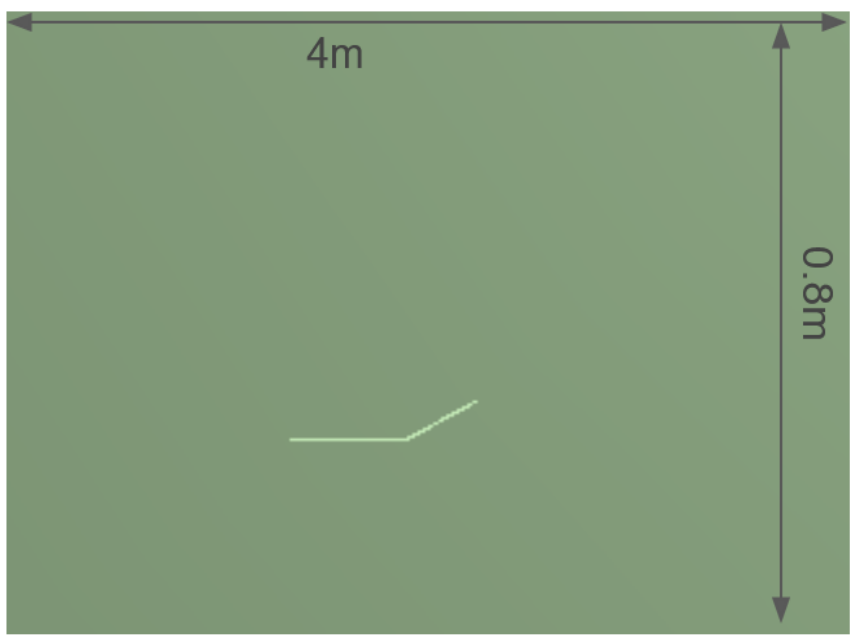

Figure 6. Fluid \& solid geometry

This 2D rectangular surface surrounds the cooling scoop and is positioned such that a majority of the area lies above the cooling scoop. This helps to ensure that the complete path of the air around and trailing off of the cooling scoop is modeled. The transmission itself was not modeled for this simulation simply because the area of interest is in the fluid dynamics of the scoop alone.

With the geometry created for each design, meshing was generated for both the fluid and cooling scoop geometry. This mesh represents finite area divisions for which calculations are made at each node by the simulation software. Adaptive meshing was turned on and mesh size was manually adjusted to be 0.02 meters. To further improve the mesh quality, a mesh refinement was applied to both the fluid and solid geometry. A close up of the generated mesh for each design is shown in Figure 7 with elements color coded by mesh quality. 

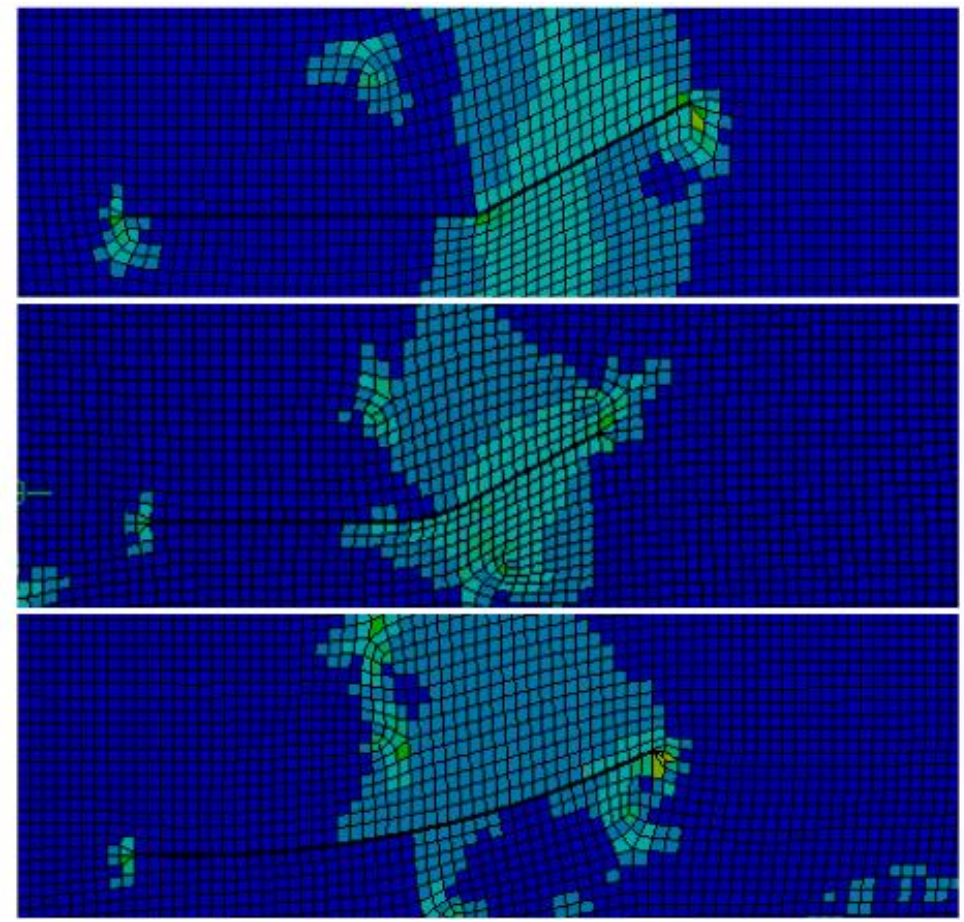

Figure 7. Refined mesh close ups for Design 1 (top), Design 2 (middle), and Design 3(bottom)

The mesh for each design contained a total of approximately 28,100 nodes and 27,800 elements each 0.02 meters in size. The generated mesh allowed for relatively accurate simulation results to be obtained without exceeding the element limit of the ANSYS license used.

In ANSYS 2D Fluent, viscous k-epsilon was selected as the model for analysis and set to be realizable with scalable wall functions. The fluid was set to standard air and the scoop was set to aluminum. Next, boundary conditions were specified. The left edge of the fluid domain was selected for the flow inlet with a velocity set to 24.6 meters per second. The right fluid domain edge was set as a pressure outlet. The horizontal edges of the fluid domain and cooling scoop geometry were set as walls. Under solution methods, green gauss node based gradient was selected and second order was selected for turbulent kinetic energy and turbulent dissipation rate. Under residual monitors, all residuals were increased in precision to 0.000001 . The fluent simulation was then initialized and the number of iterations for the solver was set to 10 and the solver was run multiple times gradually increasing the number of iterations until convergence was reached at roughly 600 iterations. This process was repeated for each of the three cooling scoop designs.

In post processing, a velocity contour map and velocity streamline plot for each design were generated. The velocity was probed at the cooling scoop entrance and exit. The exit velocity probe was positioned at the theoretical transmission location, $7 \mathrm{~cm}$ above and $10 \mathrm{~cm}$ to the right of the cooling scoop tip. In ANSYS, this probe was set to the equivalent location within the global coordinate system at $\mathrm{x}=0.5$ and $\mathrm{y}=0.15$. This was kept constant for each design.

The simulated exit velocity was used to calculate the approximate convection coefficient for air using Equation (2). To calculate the equivalent heat transfer rate, a known exposed surface area of the transmission is required, as seen in equation (1). Since the exposed surface area varies from vehicle to vehicle, it would be more beneficial and practical to calculate the heat transfer rate per unit area. This allows for a heat transfer performance value that is independent of vehicle transmission geometry. The heat transfer rate per unit area or heat flux, q" was calculated using Equation (3).

$$
q^{\prime \prime}=h_{c, \text { air }} * \Delta T, \Delta T=\left(T_{s}-T_{\infty}\right)
$$

The heat flux is equal to the convection coefficient of the ambient air multiplied by the temperature differential. For each of the cooling scoop designs, the ambient air was assumed to be at standard temperature, 25 degrees Celsius, equal to 299.15 Kelvin. The transmission temperature was assumed to be at normal operating temperature of 79.4 degrees Celsius, equal to 352.6 Kelvin. This 
gives an assumed temperature differential of 53.45 Kelvin. Using the assumed temperature differential, the equivalent heat flux was calculated for each of the three simulated designs.

A parametric study was then conducted in 2D for the optimal design based on the resulting heat flux values. The angle of attack for the diagonal component of this design was adjusted from 20 to 60 degrees with an interval of 5 degrees. The angle of attack, $\theta$, for the cooling scoop is a measure of the angle between the vertical member and horizontal datum, as shown in Figure 8.

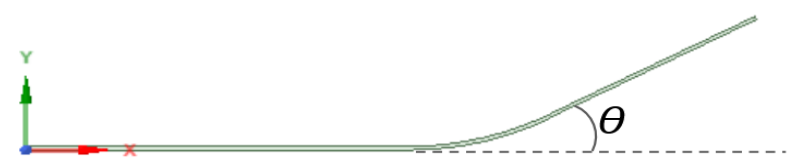

Figure 8. Angle of attack, $\theta$

The angle of attack range was manually entered as the input for the parametric study. Following the same procedures previously described, a velocity probe was again positioned at the exit of the scoop. This probe was set as an output parameter for this study. The approximate convection coefficient and equivalent heat flux were then calculated for each outputted velocity value.

Lastly, the previously mentioned 3D analysis for the cooling system was conducted. Design 1 was modeled in 3D and simulated in ANSYS with similar conditions to the 2D analysis. The 3D model incorporated a modification of design 1 with an added width of $30 \mathrm{~cm}$ and sidewalls at each edge. A 3D enclosure scaled to be $150 \%$ larger than the cooling scoop model was created to simulate the fluid geometry. The geometry for this 3D design with fluid enclosure is shown in Figure 9.

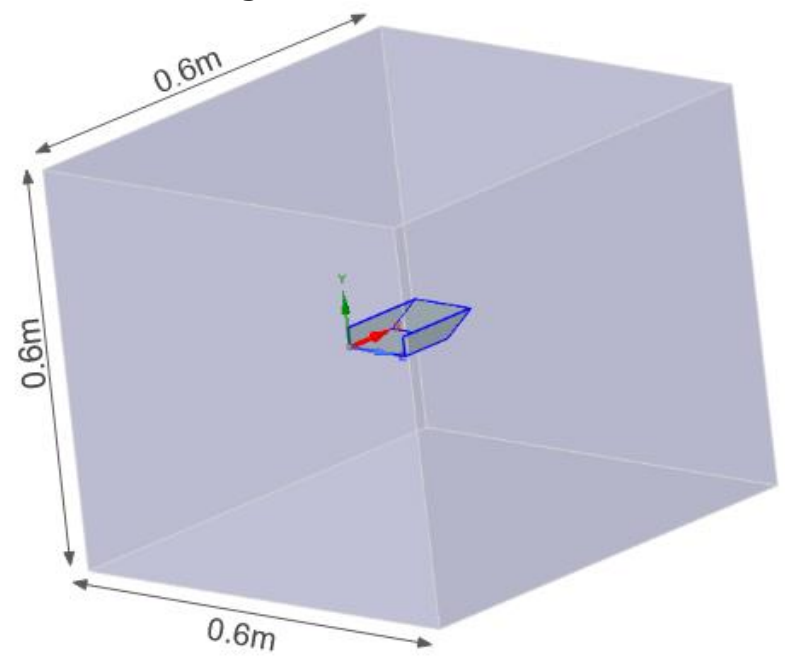

Figure 9. 3D Design 1 scoop and fluid enclosure geometry

To create the mesh for this 3D simulation, a body refinement was applied to both the enclosure and cooling scoop and mesh size was adjusted to 0.16 meters. The generated mesh for the 3D geometry is shown in Figure 10.

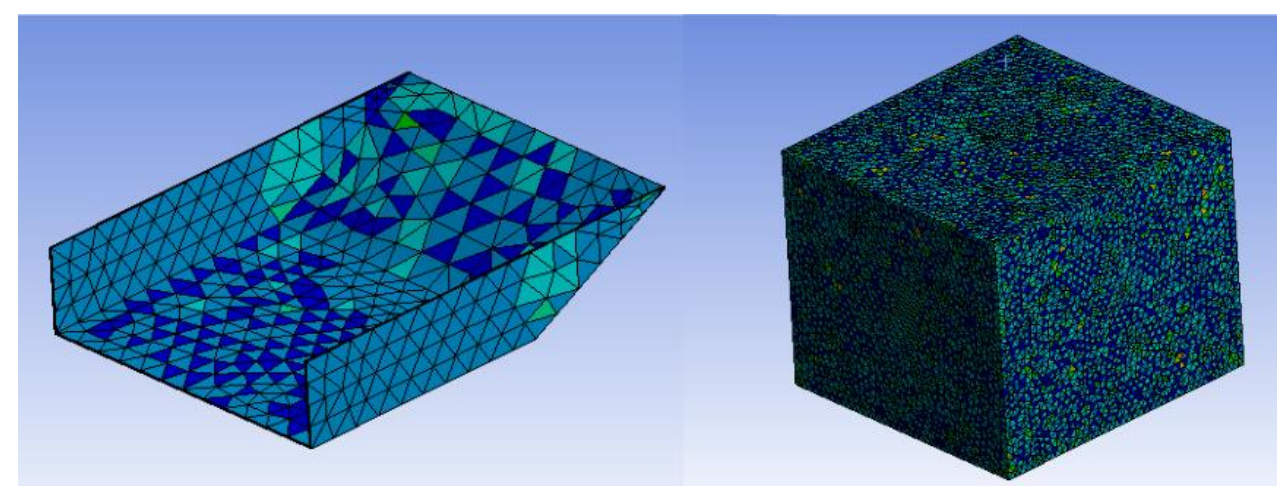

Figure 10. 3D Design 1 scoop and fluid enclosure mesh 
The total number of nodes generated was 4028. The total number of elements generated was 21260 . As opposed to the 2D simulation, the elements in this simulation are generated as finite fluid volumes for the enclosure and finite surface areas of the scoop. Nearly identical procedures were followed in ANSYS Fluent for this 3D case with the only changes consisting of applying entire faces rather than 2D edges for inlet and outlet conditions.

\section{Results and Discussions}

The resulting velocity contour maps and streamline plots generated for each of the three 2D designs are shown in Figure 11.

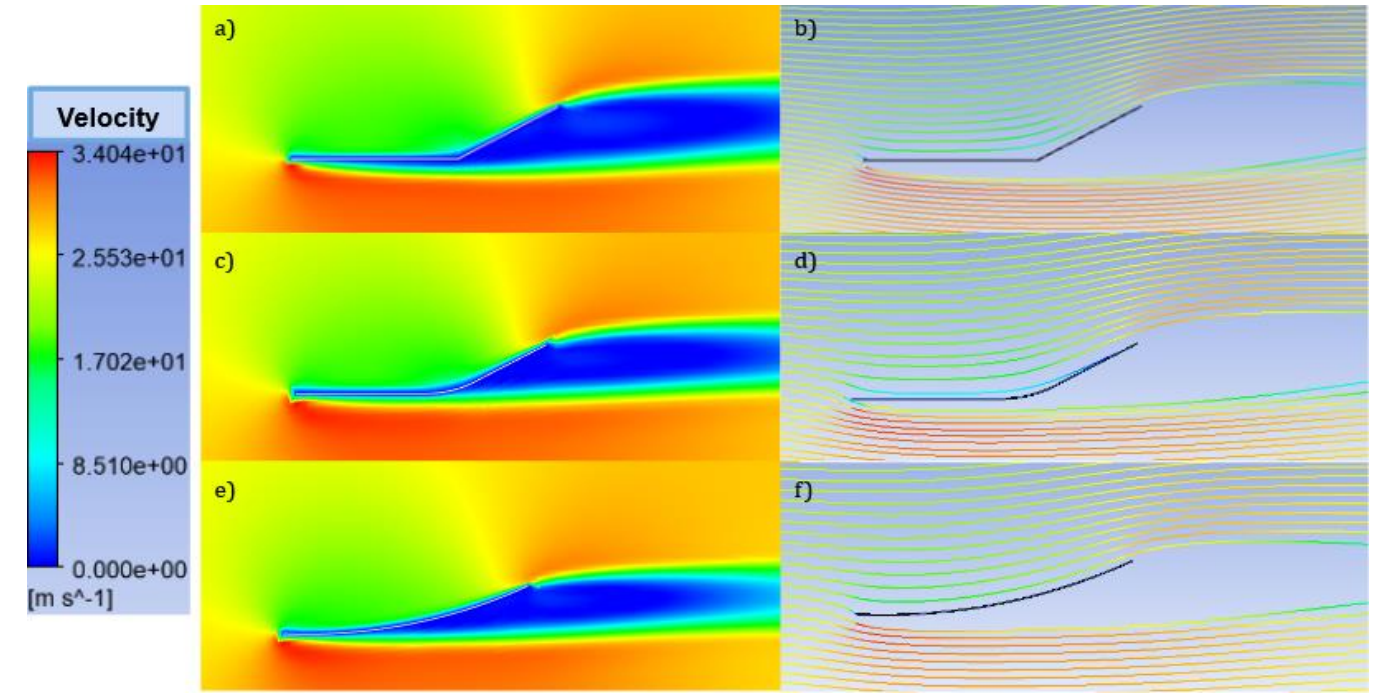

Figure 11. (a) Design 1 Velocity Contour Map; (b) Design 1 Velocity Streamline Plot;

(c) Design 2 Velocity Contour Map; (d) Design 2 Velocity Streamline Plot;

(e) Design 3 Velocity Contour Map; (f) Design 3 Velocity Streamline Plot

From the velocity contour maps and streamline plots, the greatest velocity is present below the cooling scoop and just above the right tip of the schooling scoop. It is difficult to visually see the difference in these designs from these plots, thus specific values were probed from these plots for better comparison. Using the probing method previously described, the velocity at the entrance of the scoop and at the theoretical transmission location just above the scoop exit were probed for each design for comparison. The increase in velocity from entrance to exit for each design was calculated from the respective velocity differences. Probed velocities and equivalent velocity increase for each 2D design are shown in Table 1.

Table 1. Probed fluid velocities

\begin{tabular}{|c|c|c|c|}
\hline \multirow{2}{*}{ Value } & \multicolumn{3}{|c|}{ Design } \\
\cline { 2 - 4 } & Design 1 & Design 2 & Design 3 \\
\hline $\begin{array}{c}\text { Probed Velocity at } \\
\text { Scoop Exit (m/s) }\end{array}$ & 28.2585 & 28.4476 & 28.0542 \\
\hline $\begin{array}{c}\text { Probed Velocity at } \\
\text { Scoop Entrance } \\
(\mathrm{m} / \mathrm{s})\end{array}$ & 20.9011 & 21.0239 & 21.2239 \\
\hline $\begin{array}{c}\text { Velocity Increase } \\
(\mathrm{m} / \mathrm{s})\end{array}$ & 7.3574 & 7.4237 & 6.8303 \\
\hline \hline
\end{tabular}

As seen in Table 1, design 2 yielded both the greatest velocity at the scoop exit and the greatest increase in velocity from the entrance to the exit, with design 1 yielding the second greatest of each value, and design 3 yielding the smallest of each.

The resulting cooling scoop exit velocities for each design were used to calculate the approximate convection coefficients and equivalent heat flux values. The calculated values are shown for each of the three 2D designs in Table 2. 
Table 2. 2D Scoop exit velocity, convection coefficient, and heat flux

\begin{tabular}{|c|c|c|c|}
\hline Design & $\begin{array}{c}\text { Probed Velocity } \\
\text { at Scoop Exit } \\
(\mathrm{m} / \mathrm{s})\end{array}$ & $\begin{array}{c}\text { Approximated } \\
\text { Convection } \\
\text { Coefficient, hc } \\
\left(\mathrm{W} / \mathrm{m}^{\wedge} 2^{\star} \mathrm{K}\right)\end{array}$ & $\begin{array}{c}\text { Calculated } \\
\text { Equivalent Heat } \\
\text { Flux, q" } \\
\left(\mathrm{kW} / \mathrm{m}^{\wedge} 2\right)\end{array}$ \\
\hline Design 1 & 28.259 & 188.09 & 10.053 \\
\hline Design 2 & 28.448 & 189.08 & 10.106 \\
\hline Design 3 & 28.054 & 187.02 & 9.996 \\
\hline
\end{tabular}

Again, design 2 resulted in the greatest exit air velocity directed towards the transmission. Hence, design 2 produced the greatest approximated convection coefficient of $189.08 \mathrm{~W} / \mathrm{m}^{\wedge} 2^{*} \mathrm{~K}$ and greatest equivalent heat flux of $10.106 \mathrm{~kW} / \mathrm{m}^{\wedge} 2$, making it the most optimal design for greatest cooling potential.

Based on these results, design 2 was chosen for the 2D parametric study on scoop angle of attack. The 2D parametric study results including hand calculated approximate convection coefficients and equivalent heat flux values are shown in Table 3.

Table 3. 2D Parametric study results for Design 2

\begin{tabular}{|c|c|c|c|}
\hline $\begin{array}{c}\text { Angle } \\
\text { of } \\
\text { Attack } \\
\text { (degrees) }\end{array}$ & $\begin{array}{c}\text { Probed Velocity } \\
\text { at Scoop Exit } \\
(\mathrm{m} / \mathrm{s})\end{array}$ & $\begin{array}{c}\text { Approximated } \\
\text { Convection } \\
\text { Coefficient, hc } \\
\left(\mathrm{W} / \mathrm{m}^{\wedge} \mathbf{2}^{\star} \mathrm{K}\right)\end{array}$ & $\begin{array}{c}\text { Calculated } \\
\text { Equivalent Heat } \\
\text { Flux, q" } \\
\left(\mathrm{kW} / \mathrm{m}^{\wedge} \text { 2) }\right.\end{array}$ \\
\hline 20 & 26.365 & 178.07 & 9.518 \\
\hline 25 & 27.308 & 183.08 & 9.786 \\
\hline 30 & 28.376 & 188.71 & 10.086 \\
\hline 35 & 30.281 & 198.63 & 10.617 \\
\hline 40 & 31.672 & 205.80 & 11.000 \\
\hline 45 & 32.184 & 208.42 & 11.140 \\
\hline 50 & 32.187 & 208.43 & 11.141 \\
\hline 55 & 32.188 & 208.44 & 11.141 \\
\hline 60 & 31.122 & 202.97 & 10.849 \\
\hline
\end{tabular}

The general trend shown in Table 3 suggests that increasing the angle of attack results in increased exit air velocity. Specifically, the velocity increases relatively steadily up to 45 degrees where the value remains nearly unchanged until 60 degrees where it begins to decrease. Using angle of attack and equivalent heat flux values in Table 3, a plot of the angle of attack in degrees versus the calculated equivalent heat flux in kilowatts per square meter was generated. This plot is shown in Figure 12 .

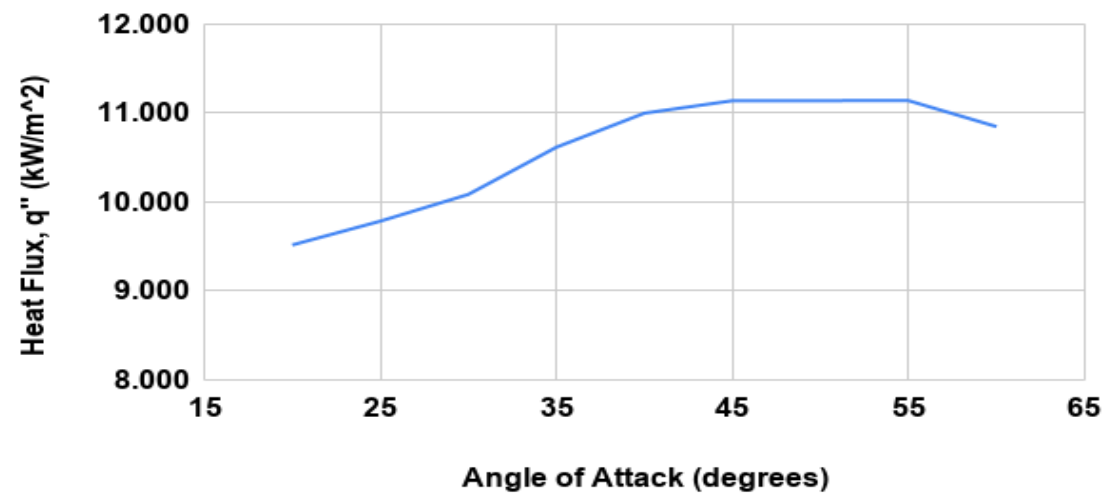

Figure 12. Angle of attack vs. heat flux, Design 2

In Figure 12, the change in equivalent heat flux with increasing angle of attack is seen. The heat flux increases from 20 to 45 degrees where it then plateaus until decreasing again at 60 degrees. The specific values at 45, 50, 55 degrees from Table 3 show little variation, all of which generating an approximate heat flux of $11.14 \mathrm{~kW} / \mathrm{m}^{\wedge} 2$. This suggests that any angle within this range of $45-55$ may be adequate for achieving maximum heat flux. 
From the 3D simulation conducted for design 1, a velocity streamline plot was generated for the 3D design 1 scoop model. The 3D streamline plot for design 1 is shown in Figure 13 below.

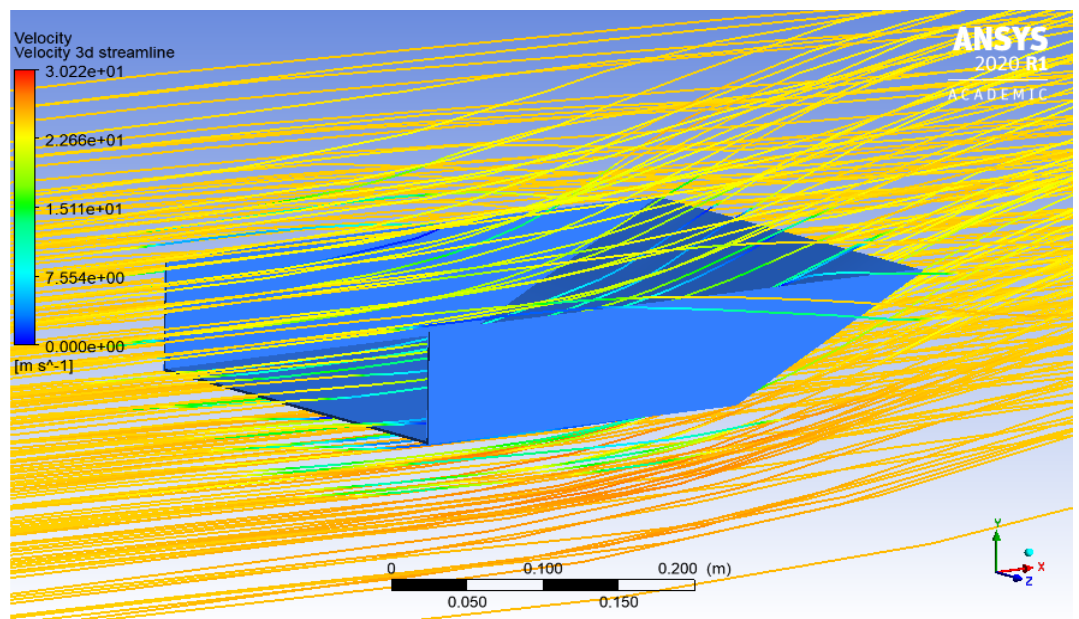

Figure 13. Design 13D cooling scoop streamline plot

The air flow around the cooling scoop of design 1 appears relatively similar to that of the 2 dimensional design 1 model where air is shown to be directed upward when exiting the scoop tip. It is important to notice the change in fluid flow from center to outer edges of the scoop exit tip shown to the right in Figure 13. The streamlines here help to determine the optimal location of cooling scoop placement with respect to the transmission. Optimal positioning for highest heat flux potential exits where the majority of exiting air velocity streamlines are directed. This location exists with the cooling scoop positioned symmetrically centered below the transmission with the scoop exit tip at the beginning of the transmission bell housing. A 3D turbulence contour map was also generated showing turbulent intensity across the cooling scoop of design 1, shown in Figure 14.

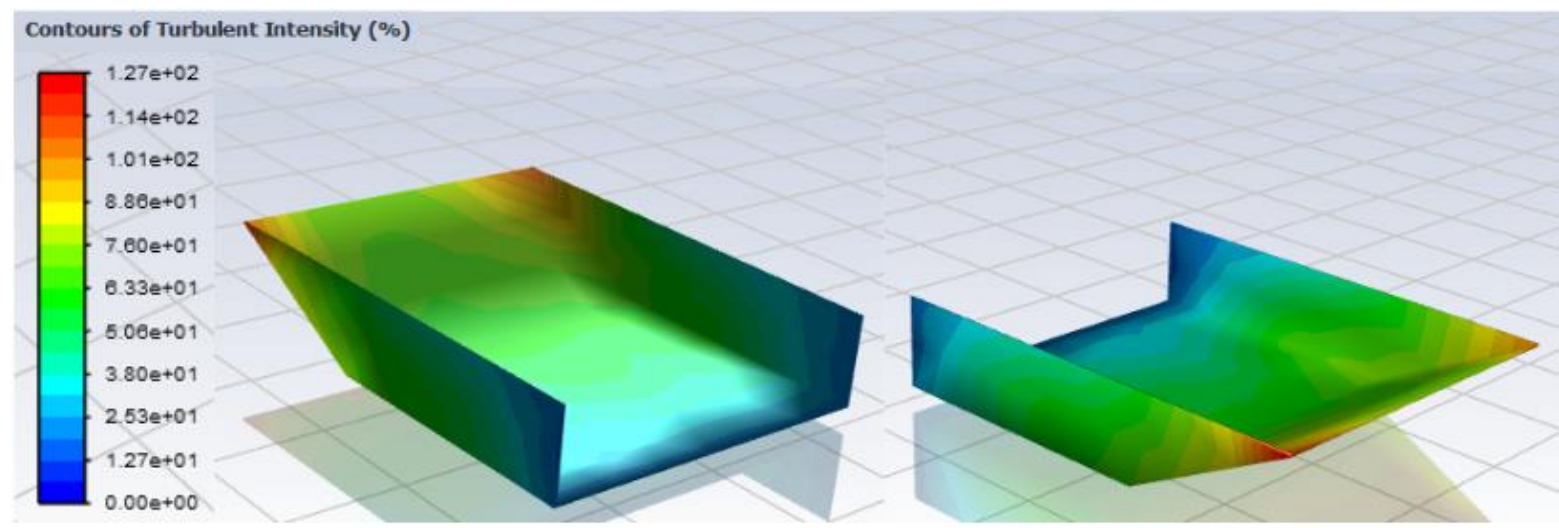

Figure 14. Design $13 \mathrm{D}$ cooling scoop turbulent intensity contour map; front and back views

The turbulent flow induced by the cooling scoop is another important factor in the cooling performance of the 3D physical model. As shown in Figure 14, flow exiting the tip at the two corners is relatively turbulent. Convective heat transfer benefits from turbulent flow because as the Reynolds number of a fluid increases, the Nusselt number increases [12] [13]. The Nusselt number quantifies the ratio of convective heat transfer to conductive heat transfer at a boundary. Hence, a high Nusselt number is beneficial for improved convective heat transfer. When only laminar flow is present at a lower Reynolds number, an insulating layer is formed reducing the rate of heat transfer. When turbulent flow occurs at higher Reynolds number, the insulating layer is broken up and the random motion of the turbulent flow assists in more rapid heat transfer. Perhaps future research could investigate alternative geometries with added components to induce turbulent flow at the center of the cooling scoop where the majority of airflow exists. 


\section{Conclusions}

The use of forced convection for cooling of a vehicle transmission was investigated for three compact cooling scoop designs. All three cooling scoop designs successfully increased airflow to the transmission, with design 2 resulting in the highest air velocity increase of $7.424 \mathrm{~m} / \mathrm{s}$ and greatest overall velocity directed at the transmission of $28.448 \mathrm{~m} / \mathrm{s}$. This yielded an approximated convection coefficient of $189.08 \mathrm{~W} / \mathrm{m}^{\wedge} 2^{*} \mathrm{~K}$ and equivalent heat flux of $10.106 \mathrm{~kW} / \mathrm{m}^{\wedge} 2$. Hence, design 2 was chosen as the most optimal design geometry.

To further optimize design 2, a parametric study was conducted to determine the optimal angle of attack to be implemented into the design. From Figure 12, the optimal angle of attack is achieved between 45 and 55 degrees, where the highest equivalent heat flux of $11.14 \mathrm{~kW} / \mathrm{m}^{\wedge} 2$ was generated. In an attempt to reduce vehicle drag, 45 degrees may be the most optimal angle of attack since it is the most streamline within this optimal angle of attack range.

Lastly, a 3 dimensional simulation was run for design 1 to check for any issues that may not be visible in the 2 dimensional simulation. As determined from Figure 13, optimal flow is generated with the cooling scoop positioned symmetrically centered below the transmission with the scoop exit tip at the beginning of the transmission bell housing. Future designs would benefit from purposely induced turbulence at the central exit of the cooling scoop tip resulting in higher potential heat transfer rate.

\section{References}

[1] D. Young, \& M. Barnholt, "Forced Convection Axle Cooling," United States Patent 6036615, Mar. 14, 2000.

[2] K. Miller, T. Leone, \& A. Dudar, "Powertrain Fault Management," United States Patent 10401853 B2, Sep. 3, 2019.

[3] A. Gerling, H. Hengstler, \& H. Hoffstatter, "Method for Reducing the Thermal Load on an Automatic Transmission for a Motor Vehicle in an Emergency Operating Mode," United States Patent 6632157, Oct. 14, 2003.

[4] Toyota Sienna Owners Guides and Service Manuals. Toyota Sienna Service Manual: Transmission Fluid Temperature Sensor "A" [Online]. [cited 2021 Jan 14]. Available from: http://www.tsienna.net/transmission_fluid_temperature_sensor_quot_a_quot_-779.html

[5] J. P. Chiou, "Air-Cooled vs Liquid-Cooled Transmission Oil Cooler for Heavy-Duty Vehicles," SAE Technical Paper Series, 1978; vol. 86: pp. 2677-2694.

[6] A. Stine, "Transmission Cooling System," United States Patent 5678461, Oct. 21, 1997.

[7] S. Joshi, "Oil Cooler," United States Patent 4945981, Aug. 7, 1990.

[8] A. Belhocine, \& W.Z.W Omar, "CFD analysis of the brake disc and the wheel house through air flow: Predictions of Surface heat transfer coefficients (STHC) during braking operation," Journal of Mech. Sci. and Tech., [Online], Sept 2017 [cited 2021 Jan 14]; vol. 32: pp. 481-490, Available from: https://link.springer.com/article/10.1007\%2Fs12206-017-1249-z

[9] M. Evans, "Transmission Cooling Device," United States Patent 4848453, Jul. 18, 1989.

[10] W. Minnichsoffer, \& J. Parsons, “Cooling Duct,” United States Patent 0088289, Mar. 19, 2020.

[11] A. Ferrantelli, P. Melois, \& M. Viljanen, "Sensitivity Studies of Heat Transfer: Forced Convection Across a Cylindrical Pipe and Duct Flow," Aalto University, Department of Civil and Structural Engineering, [Online], August 2013 [cited 2020 Dec 20], Available from: https://www.researchgate.net/publication/255787211_Sensitivity_studies_of_heat_transfer_forc ed_convection_across_a_cylindrical_pipe_and_duct_flow.

[12] L. Qiang, \& X. Yimin, "Convective heat transfer and flow characteristics of Cu-water nanofluid," Science in China, [Online], August 2002 [cited 2021 Jan 14]; vol. 45 (No. 4): pp. 409-416, Available from: https://link.springer.com/article/10.1360/02ye9047

[13] A.R. Sajadi, \& M.H. Kazemi, "Investigation of turbulent convective heat transfer and pressure drop of TiO2/water nanofluid in circular tube," Int. Com. in Heat and Mass Transfer, [Online], July 2011 [cited 2021 Jan 14]; vol. 38: pp. 1474-1478, Available from: https://www.journals.elsevier.com/international-communications-in-heat-and-mass-transfer. 